\title{
An Agent-Based Simulation for Investigating the Impact of Stereotypes on Task-Oriented Group Formation
}

\author{
Mahsa Maghami and Gita Sukthankar \\ Department of EECS \\ University of Central Florida \\ Orlando, FL \\ mmaghami@cs.ucf.edu, gitars@eecs.ucf.edu
}

\begin{abstract}
In this paper, we introduce an agent-based simulation for investigating the impact of social factors on the formation and evolution of task-oriented groups. Task-oriented groups are created explicitly to perform a task, and all members derive benefits from task completion. However, even in cases when all group members act in a way that is locally optimal for task completion, social forces that have mild effects on choice of associates can have a measurable impact on task completion performance. In this paper, we show how our simulation can be used to model the impact of stereotypes on group formation. In our simulation, stereotypes are based on observable features, learned from prior experience, and only affect an agent's link formation preferences. Even without assuming stereotypes affect the agents' willingness or ability to complete tasks, the long-term modifications that stereotypes have on the agents' social network impair the agents' ability to form groups with sufficient diversity of skills, as compared to agents who form links randomly. An interesting finding is that this effect holds even in cases where stereotype preference and skill existence are completely uncorrelated.
\end{abstract}

Keywords: Group formation, Multi-agent social simulations, Stereotypes

\section{Introduction}

Group membership influences many aspects of our lives, including our self-identities, activities, and associates; it affects not only what we do and who we do it with, but what we think of ourselves and the people around us. It can also give rise to stereotypic thinking in which group differences are magnified and importance of individual variations are discounted. Thinking categorically about people simplifies and streamlines the person perception process [12], facilitating information processing by allowing the perceiver to rely on previously stored knowledge in place of incoming information [11]. Whereas a variety of stereotypes are based on real group differences (e.g., cultural stereotypes about food preferences), stereotypes based on relatively enduring characteristics, such as race, religion, and gender, have an enormous potential for error [11] and can give rise to performance impairments [13]. We hypothesize that when stereotypes are formed independent of real group differences, it can result in negative effects for the collective system. However, studying the long-term effects of stereotypes can be difficult, especially to quantify the effects over a population rather than an individual. In this paper, 
we describe an agent-based simulation for evaluating the impact of stereotypes on the performance of task-oriented groups. Understanding the role that stereotypes play in group formation can refine existing theory while providing insight into the efficacy of methods for combating the adverse effects of stereotypes on behavior and decisionmaking.

We base our simulation on a model of multi-agent team formation [6] since taskoriented groups share many characteristics with teams, although lacking in shared training experience. In our simulation, the population of agents is connected by a social network that is locally updated over time by unoccupied agents depending on their preferences. Stereotypes are represented as an acquired preference model based on prior experience and observable agent features. In multi-agent environments, stereotypes have been used to provide faster modeling of other agents $[4,5]$ and to bootstrap the trust evaluation of unknown agents [2]. In contrast, we examine the case of non-informative stereotypes; stereotypes affect the agents' preferences for forming social attachments but do not affect the agents' willingness or ability to cooperate with other agents.

\section{Problem Statement}

To explore the impact of stereotype on group formation and network evolution, we have selected a simple multi-agent system model first introduced by Gaston and desJardins [6] and used in $[7,8]$ to describe team formation. Since task-oriented groups are similar to teams, this is a reasonable method for modeling the task performance of group behavior on shared utility tasks in absence of stereotypes. In this model, there is a population of $N$ agents represented by the set $A=\left\{a_{1}, \ldots, a_{N}\right\}$. Each agent can be considered as a unique node in the social network and the connection between the agents is modeled by an adjacency matrix $E$, where $e_{i j}=1$ indicates an undirected edge between agent $a_{i}$ and $a_{j}$ and the degree of agent $a_{i}$ is defined as $d_{i}=\sum_{a_{j} \subseteq A} e_{i j}$. Each agent is assigned a single skill given by $\sigma_{i} \in[1, \sigma]$ where $\sigma$ is the number of available skills. Accomplishing each task requires a coalition of agents with the appropriate skills. Tasks are globally advertised for $\gamma$ time steps at fixed intervals $\mu$. Each task, $T_{k}$, has a size requirement, $\left|T_{k}\right|$, and a $\left|T_{k}\right|$-dimensional vector of required skills, $R_{T_{k}}$, which are selected uniformly from $[1, \sigma]$. When a coalition has formed with the full complement of skills required for the task, it takes $\alpha$ time steps for the group to complete the task.

\subsection{Group Formation}

In the no stereotype case, we simply follow the group formation algorithm used to allocate agents to teams in [7].

During the team formation process, each agent, $a_{i}$, can be in one of three states, $s_{i}$, UNCOMMITTED, COMMITTED, or ACTIVE. An agent in the UNCOMMITTED state has not been assigned to any task. An agent in the COMMITTED state has been assigned to a task but is still waiting for the enough agents with the right skills to join the group. Finally, an ACTIVE agent is currently working on a task with a complete group possessing the right complement of skills. 
On each iteration, agents are updated in random order. UNCOMMITTED agents have the opportunity to adapt their local connectivity (with probability of $P_{i}$ ) or can attempt to join a group. If a task currently has no other agents committed to it, an agent can initiate a group with a probability that is proportional to the number of its immediate UNCOMMITTED neighbors defined as follows:

$$
I P_{i}=\frac{\sum_{a_{j} \subseteq A} e_{i j} I\left(s_{i}, \text { UNCOMMITTED }\right)}{\sum_{a_{j} \subseteq A} e_{i j}},
$$

where $I(x, y)=1$ when $x=y$ and 0 otherwise.

Agents are only eligible to join task-oriented groups in their local neighborhood, where this is at least one link between the agent and the group members. The algorithm used by an agent to initiate or join a group is presented in Algorithm 1.

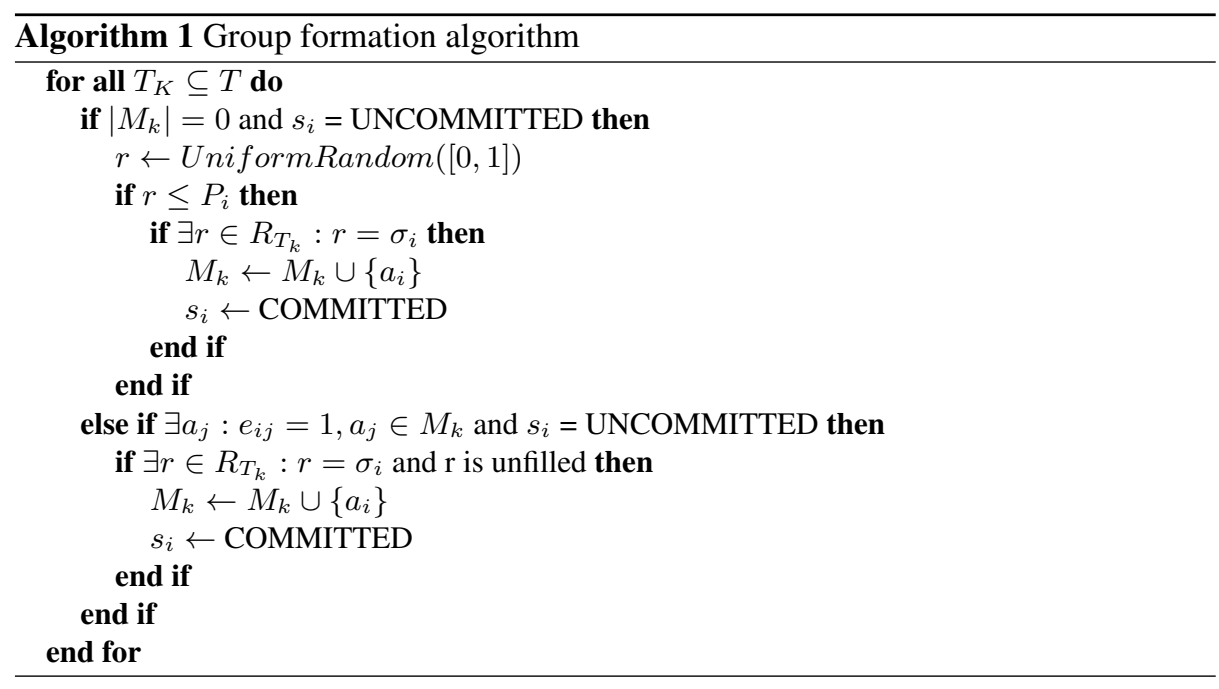

\subsection{Network Adaptation}

To adapt the network structure, the agents modify their local connectivity based on the notion of preferential attachment [1]. Therefore, the probability of connecting to a given node is proportional to that node's degree. As mentioned before, at each iteration the agent can opt to adapt its connectivity, with probability $P_{i}$. Modifying its local connectivity does not increase the degree of the initiating agent since the agent severs one of its existing connections at random and forms a new connection.

To form a new connection, an agent considers the set of its neighbors' neighbors designated as $N_{i}^{2}=\left\{a_{m}: e_{i j}=1, e_{j m}=1, e_{i m}=0, m \neq i\right\}$. The adapting agent, $a_{i}$, selects a target agent, $a_{j} \subseteq N_{i}^{2}$, to link to based on the following probability distribution:

$$
P\left(a_{i} \longrightarrow a_{j}\right)=\frac{d_{j}}{\sum_{a_{l} \subseteq N_{i}^{2}} d_{l}},
$$


where $d$ is the degree of agents.

The results in [7] and [8] show that this simple algorithm can be used to adapt a wide variety of random network topologies to produce networks that are efficient at information propagation and result in scale-free networks similar to those observed in human societies. For the non-stereotype group formation condition, we did not observe any differences between random attachment and preferential attachment.

\section{Learning the Stereotype Model}

As noted in a review of the stereotype literature [11], stereotypes are beliefs about the members of a group according to their attributes and features. It has been shown that the stereotypes operate as a source of expectancies about what a group as a whole is like as well as what attributes individual group members are likely to possess [9]. Stereotype influences can be viewed as a judgment about the members of a specific group based on relatively enduring characteristics rather than their real characteristics.

Here, we do not attempt to capture the rich cognitive notions of stereotype formation as it applies to humans, but rather view a stereotype as a function $\mathcal{F}: \overrightarrow{\mathrm{V}} \longrightarrow S$, mapping a feature vector of agents, $\vec{V}$, to a stereotypical impression of agents in forming friendships, $S$, which we will designate as the stereotype value judgment. This value represents the agents' judgments on other groups and is based on observable features rather than skills or prior task performance.

In most contexts, humans possess two types of information about others: 1) information about the individual's attributes and 2) the person's long-term membership in stereotyped groups [9]. Therefore, to learn the stereotype model, the simulation offers these two sources of information, $\vec{V}$ and its corresponding $S$ which are related to the agents' group membership, for a specific period of time. In our simulation, this initial period lasts for $I$ time steps and helps the collaborating agents gain experience about the attributes of different groups of agents. Note that membership in these groups is permanent and not related to the agent's history of participation in short-term task-oriented groups.

In our work, we propose that each agent, $a_{i}$, can use linear regression to build its own function, $\mathcal{F}_{i}$, and to estimate the stereotype value of another agent, $a_{j}$, according to the observable features $\overrightarrow{\mathrm{V}}_{\mathrm{j}}$. After the initial period, $I$ time steps, the estimated stereotype value of agent $a_{j}$ by agent $a_{i}$ will be calculated as $\hat{S_{i j}}=\mathcal{F}_{i}\left(\overrightarrow{\mathrm{V}_{j}}\right)$.

In our model, this stereotype value judgment affects the connection of agents during the network adaptation phase, as we will describe in the following section.

\subsection{Network Adaptation with Stereotype Value Judgments}

In the stereotype case, the group formation algorithm is the same as described in Algorithm 1 but the network adaptation is based on the learned stereotype. If an agent decides to adapt its local network, again with probability $P_{i}$, it will do so based on its own stereotype model. To adapt the local connectivity network, each agent uses its learned model to make stereotype value judgment on other neighboring agents. This network adaptation process consists of selecting a link to sever and forming a new link. 
Specifically, the agent $a_{i}$ first searches for its immediate neighbor that has the lowest stereotype value judgment, $a_{j}$, and severs that link. The agent then searches for a new agent as a target for link formation. To form this link, it searches its immediate neighbors and the neighbors of neighbors. First the agent selects the neighbor with the highest stereotype value judgment, $a_{m}$, for a referral as this agent is likely to be a popular agent in its neighborhood. Then the adapting agent, $a_{i}$, will establish a new connection with $a_{n}$, one of the most popular neighbors of $a_{m}$, assuming that it is not already connected.

$$
a_{n}=\arg _{a_{k} \in A, e_{i k}=0} \max \hat{S_{i k}} .
$$

Note that all of these selections are the result of the stereotype value judgment model that agent $a_{i}$ has about the other agents in its neighborhood.

\section{Evaluation}

\subsection{Experimental Setup}

We conducted a set of simulation experiments to evaluate the effects of stereotype value judgments on group formation in a simulated society of agents. The parameters of the group formation model for all the runs are summarized in Table 1. In task generation, each task is created with a random number of components less than or equal to ten and a vector of uniformly-distributed skill requirements. To generate the agent society, each agent is assigned a specific skill, a feature vector, and a class label. The agents' skills are randomly generated from available skills. Inspired by [2], four different long-lasting groups with different feature vector distributions are used as the basis for stereotype value judgments. Agents are assigned a six-dimensional feature vector, with each dimension representing an observable attribute, and a hidden stereotype value judgment drawn from Gaussian distribution assigned to the group. Table 2 shows the mean and standard deviations of the Gaussian distributions and the observable feature vector assigned to each group. The observable feature vectors are slightly noisy. To indicate the existence of an attribute, a random number is selected from distribution $N(0.9,0.05)$ to be close to 1 and to indicate the lack of an attribute this number is selected from distribution $N(0.1,0.05)$ to be close to zero. During the initial training period, agents are allowed to observe the hidden stereotype value judgment to learn the classifier. During the rest of the simulation these values are estimated by the agent's learned model.

In these experiments all the runs start with a random geometric graph (RGG) as the initial network topology among the agents. A RGG is generated by randomly locating all the agents in a unit square and connecting two agents if their distance is less than or equal to a specified threshold, $d$ [3]. The random network we generated is a modified version of the RGG, proposed by [7]. In this version the $d$ is selected as a minimal distance among the agents to guarantee that all the agents have at least one link to other agents.

When the initial network is generated, the group formation will run for an initial period with no adaptation. During these initial steps, the agents can form groups and participate in task completion to gain some experiences about other agents. Results are based on the average of 10 different runs with a different initial network for each run. 
(a) Experimental parameters

\begin{tabular}{ccl}
\hline Parameter & Value & Descriptions \\
\hline$N$ & 100 & Total number of agents \\
$\sigma$ & 10 & Total number of skills \\
$\gamma$ & 10 & Task advertising time steps \\
$\alpha$ & 4 & Agents active time \\
$\mu$ & 2 & Task interval \\
$|T|$ & $\max 10$ & Number of task required skills \\
$N_{\text {Iterations }}$ & 8000 & Number of iterations \\
$N_{\text {Initial }}$ & 2000 & Number of initial iterations \\
\hline
\end{tabular}

(b) Stereotype groups and feature vectors

\begin{tabular}{cccccccccc}
\hline Group & Mean Value & StDev & $f_{1}$ & $f_{2}$ & $f_{3}$ & $f_{4}$ & $f_{5}$ & $f_{6}$ \\
\hline$G 1$ & 0.9 & 0.05 & X & & & & & X \\
$G 2$ & 0.6 & 0.15 & & X & & X & & \\
$G 3$ & 0.4 & 0.15 & & & X & X & & \\
$G 4$ & 0.3 & 0.1 & & X & X & & X & \\
\hline
\end{tabular}

\subsection{Results}

Performance The performance of the system, like [7], is calculated as follows:

$$
\text { Performance }=\frac{T_{\text {SuccessfullyDone }}}{T_{\text {total }}},
$$

which is the proportion of successfully accomplished tasks over the total number of introduced tasks in the environment. Figure 1 shows the performance of system in the system with stereotypes and without stereotypes by iteration.

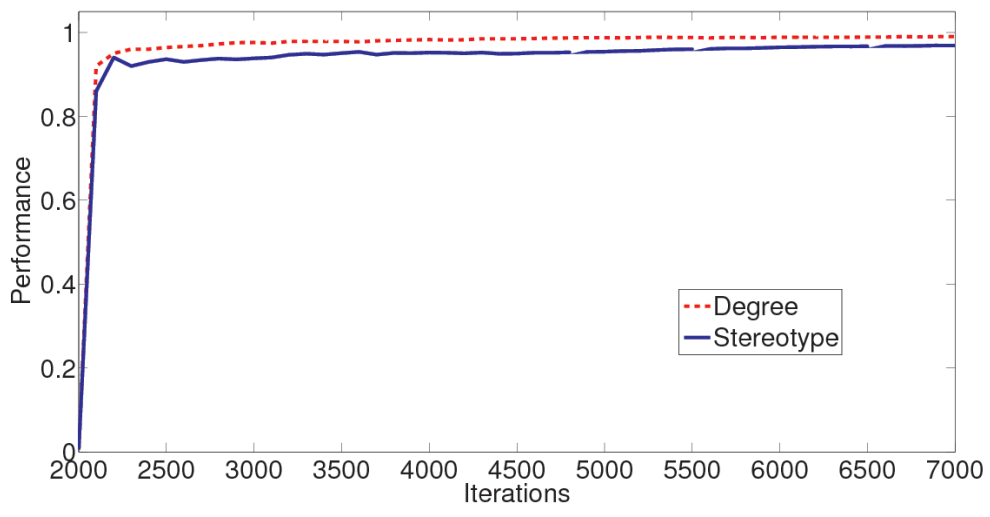

Fig. 1. The performance of task-oriented groups (with and without stereotypes) vs. the iterations

The main effect of the stereotype is to move network toward a sparse network structure with a dramatic increase in isolate nodes. This drop in performance is even more pronounced with fewer total agents.

Network Structure Here, we examine the network structure to determine the evolution of the agent society. Figure 2 shows the Fiedler-embedding [10] of networks in the final connectivity network of $N=200$ agents with and without stereotype value judgments. The degree-based strategy moves the structure toward being similar to a scale-free network whereas with stereotype value judgments the network becomes progressively more star-shaped. 

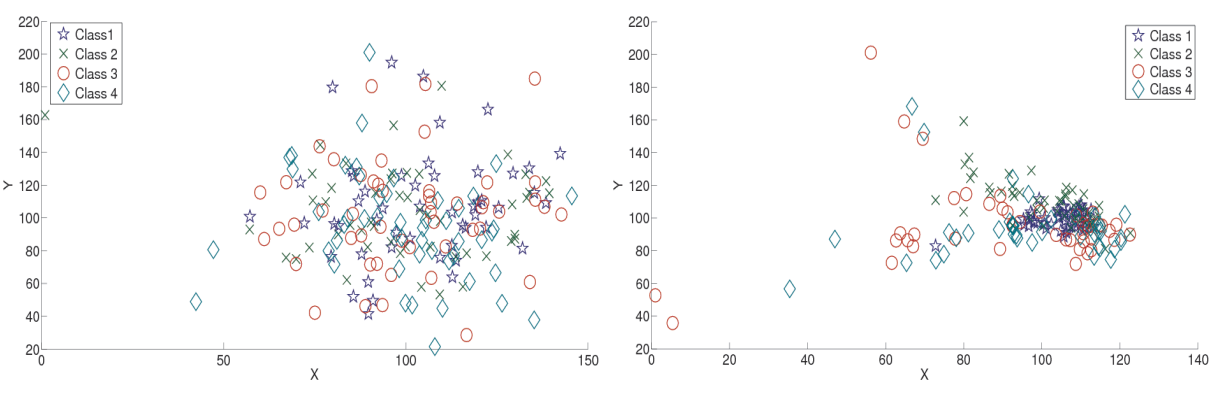

Fig. 2. Fiedler embedding of the final network structures in non-stereotype (left) and stereotype (right) based network evolution $(N=200)$

Effects of Rapid Attachment Modification Here we examine the effects of modifying the parameter $P$, the probability of updating the network, on the performance of the system, both with and without stereotypes. We varied this parameter from 0.1 to 0.9 with a step size 0.2. Figure 3 shows the performance modification during 5000 iterations in both strategies. As shown in the figure, the performance does not change significantly with $P$ values before a certain threshold. After that threshold, the performance drops dramatically, as the agents spend more time updating the network than accomplishing tasks. This threshold is dependent on the total agents and number of skills required in the environment. In both strategies, the performance has dropped by $P=0.7$ but in stereotype strategy the system drops at an earlier iteration since the information transmission efficiency of the network has been sabotaged by the stereotype-value judgments.
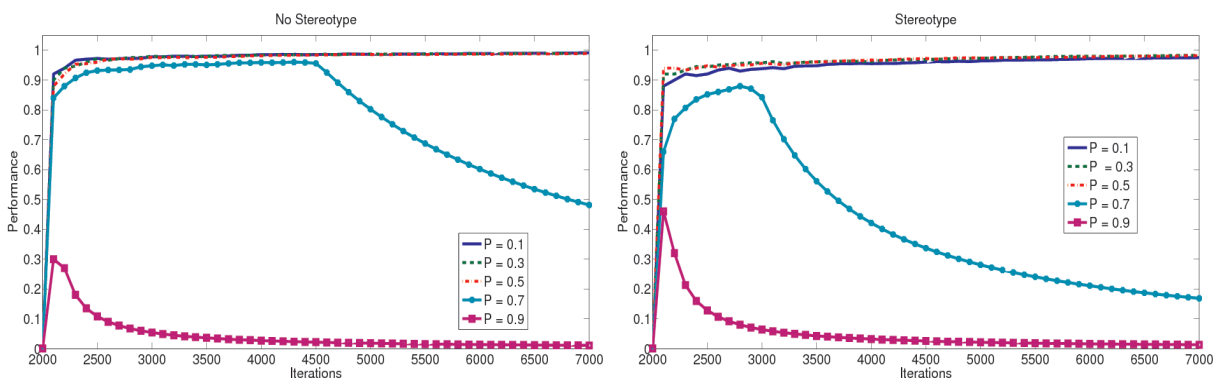

Fig. 3. The effect of the network adaptation probability, $P_{i}$

\section{Conclusion and Future Work}

In this paper we introduced an agent-based simulation for examining the effects of stereotypes on task-oriented group formation and network evolution. We demonstrate that stereotype value judgments can have a negative impact on task performance, even 
in the mild case when the agents' willingness and ability to cooperate is not impaired. By modifying the social network from which groups are formed in a systematically suboptimal way, the stereotype-driven agents eliminate the skill diversity required for successful groups by driving the network toward specific topological configurations that are ill-suited for the task. The results show that making connections with agents solely based on group membership yields a sparser network with many isolated nodes. In future work, we plan to explore the effects of different stereotype models on network topology. Additionally, we plan to incorporate EPA (Evaluation, Potency, and Accuracy) [14] style parameters into our stereotype judgment model. Due to the technical challenges of investigating the long-term effects of stereotype across populations, we suggest our agent-based simulation method is a useful tool for investigating these research questions.

\section{Acknowledgments}

This research was funded by AFOSR YIP award FA9550-09-1-0525.

\section{References}

1. Albert, R., Barabási, A.: Statistical mechanics of complex networks. Reviews of Modern Physics 74(1), 47-97 (2002)

2. Burnett, C., Norman, T., Sycara, K.: Bootstrapping trust evaluations through stereotypes. In: Proceedings of the 9th International Conference on Autonomous Agents and Multiagent Systems. pp. 241-248 (2010)

3. Dall, J., Christensen, M.: Random geometric graphs. Physical Review E 66(1), 16121 (2002)

4. Denzinger, J., Hamdan, J.: Improving modeling of other agents using tentative stereotypes and compactification of observations. In: Proceedings of Intelligent Agent Technology. pp. 106-112 (2005)

5. Denzinger, J., Hamdan, J.: Improving observation-based modeling of other agents using tentative stereotyping and compactification through kd-tree structuring. Web Intelligence and Agent Systems 4(3), 255-270 (2006)

6. Gaston, M., Simmons, J., DesJardins, M.: Adapting network structure for efficient team formation. In: Proceedings of the AAAI 2004 Fall Symposium on Artificial Multi-agent Learning (2004)

7. Gaston, M., desJardins, M.: Agent-organized networks for dynamic team formation. In: Proceedings of the International Conference on Autonomous agents and Multiagent Systems. pp. 230-237 (2005)

8. Glinton, R., Sycara, K., Scerri, P.: Agent-organized networks redux. In: Proceedings of AAAI. pp. 83-88 (2008)

9. Hamilton, D., Sherman, S., Ruvolo, C.: Stereotype-based expectancies: Effects on information pprocessing and social behavior. Journal of Social Issues 46(2), 35-60 (1990)

10. Hendrichson, B.: Latent semantic analysis and Fiedler embeddings. In: Proceedings of the Fourth Workshop on Text Mining of the Sixth SIAM International Conference on Data Mining (2006)

11. Hilton, J., Von Hippel, W.: Stereotypes. Annual Review of Psychology 47 (1996)

12. Macrae, C., Bodenhausen, G.: Social cognition: Thinking categorically about others. Annual Review of Psychology 51(1), 93-120 (2000)

13. Marx, D.M.: On the role of group membership in stereotype-based performance effects. Journal of Social and Personality Psychology Compass 3(1), 77-93 (2009)

14. Osgood, C.: The measurement of meaning. Univ of Illinois (1975) 